\title{
Integrated Weed Management in Cowpea (Vigna unguiculata (L.) Wasp.) under Rainfed Conditions
}

\author{
Pravindra Kumar ${ }^{1}$ and Raghuveer Singh ${ }^{2}$
}

${ }^{1}$ Department of Agronomy, College of Agriculture, JAU, Junagadh, Gujarat-362001, India

${ }^{2}$ ICAR-Indian Institute of Farming System Research, Modipuram, Meerut,

UttarPradesh-250110, India

*Corresponding author

\begin{tabular}{|c|c|}
\hline & A B S T R A C T \\
\hline $\begin{array}{l}\text { Ke y w or d s } \\
\text { Integrated weed } \\
\text { management, } \\
\text { Herbicides, } \\
\text { Cowpea. }\end{array}$ & $\begin{array}{l}\text { A field experiments was conducted during Kharif seasons } 2007 \text { at Junagadh Agricultural } \\
\text { University, Junagadh, Gujarat to study the effect of weed management practices on } \\
\text { cowpea under rainfed conditions. It was found that single application of herbicide or } \\
\text { cultural practices }(1 \mathrm{HW}+1 \mathrm{IC} \text { ) at } 20-25 \text { DAS not sufficient to control weed in cowpea } \\
\text { and significantly yield loss } 20-25 \% \text { recorded, which is just half of around } 45 \% \text { yield loss } \\
\text { in case no weed control measure followed. Application of (pendimethalin at } 0.5 \mathrm{~kg} \mathrm{ha}^{-1} \text { as }\end{array}$ \\
\hline Article Info & $\begin{array}{l}\mathrm{PRE}+1 \mathrm{HW}+1 \mathrm{IC} \text { at } 25-30 \mathrm{DAS} \text { ), (quizalofop- ethyl at } 0.04 \mathrm{~kg} \mathrm{ha}^{-1} \text { as POST } 20-25 \\
\mathrm{DAS}+1 \mathrm{HW}+1 \mathrm{IC} \text { at } 40-45 \mathrm{DAS} \text { ) and (imazetheyr at } 0.075 \mathrm{~kg} \mathrm{ha}^{-1} \text { as POST } 20-25\end{array}$ \\
\hline $\begin{array}{l}\text { Accepted: } \\
\text { 08 February } 2017 \\
\text { Available Online: } \\
10 \text { March } 2017\end{array}$ & $\begin{array}{l}\text { DAS + } 1 \mathrm{HW}+1 \mathrm{IC} \text { at } 40-45 \text { DAS }) \text { gave yield statistically at par with weed free plot. So } \\
\text { integrated weed management is better option and cowpea farmer can replace } 20-25 \text { DAS } \\
(1 \mathrm{HW}+1 \mathrm{IC}) \text { cultural practices with suitable herbicide to get same yield. }\end{array}$ \\
\hline
\end{tabular}

\section{Introduction}

Pulses are an integral part of many diets across the globe and they have great potential to improve human health, conserve our soils, protect the environment and contribute to global food security. The United Nations, declared 2016 as "International Year of Pulses" (IYP). India is the largest producer (25\% of global production), consumer (27\% of world consumption) and importer (14\%) of pulses in the world. Pulses account for around 20 per cent of the area under food grains and contribute around 7-10 per cent of the total food grains production in the country (Mohanty and Satyasa, 2015).
Cowpea (Vigna unguiculata (L.)) cultivated around the world primarily for seed, but also as a vegetable (for leafy greens, green pods, fresh shelled green peas, and shelled dried peas), as cover crop and for fodder Andargie et al., (2011). It is one of the important kharif pulse crop grown in the India for grain, forage, and green manure purpose and commonly known as lobia. Cowpea is also called vegetable meat because of it rich in protein 19-26 \% (average $22.5 \%$ ), carbohydrate $60.3 \%$, minerals and vitamins. 
Cowpea is a most versatile kharif as well as summer pulse, because of its smothering nature, drought tolerant character, soil restoring properties and multipurpose uses.

During rainy season the crop suffers severely due to weed infestation resulting into wide range reduction in crop yield. The critical period of crop weed competition in cowpea has been identified as 20-30 days after sowing and presence of weeds beyond this period causes severe reduction in yields Gupta et al., (2016). Hence, weed control needs to be undertaken during initial period of crop growth. Though the hand weeding is a well proven effective method of weed control, but non-availability of labour and the cost incurred in it is very high. Keeping in view the fact, the present experiment was conducted to find out suitable and cost effective weed management practice to manage weeds during the critical period of crop weed competition.

\section{Materials and Methods}

\section{Experimental site}

A field experiment was carried out in C8 block of the Instructional Farm, Department of Agronomy, Junagadh Agricultural University, Junagadh, Gujarat in kharif season 2007. Geographically this place is located at $21.50 \mathrm{~N}$ latitudes and 70.50 E longitudes with an altitude of 60 meters above the mean sea level on the western side at the foothills of Mount Girnar.

\section{Weather conditions}

This place experience the typical sub-tropical climate characterized by fairly cold and dry winter, hot and dry summer and moderately humid monsoon season. The rainy season commences in the second fortnight of June and extends up to September. The average annual rainfall is $868.6 \mathrm{~mm}$, out of which maximum rainfall occurs during the months of July and August. Partial failure of monsoon once in 3 to 4 years is very common phenomenon in this region. Winter sets in the month of November and continues till the month of February. January is the coldest month of winter. Summer season commences from the second fortnight of February and ends in the middle of June. April and May are the hottest months of summer.

\section{Experimental soil}

Soil was clayey in texture, slightly alkaline in reaction, medium in organic carbon $(0.61 \%)$ low in available nitrogen $(220.0 \mathrm{~kg} / \mathrm{ha})$ and available phosphorus (18.9 $\mathrm{kg} / \mathrm{ha})$, whereas high in available potassium $(378.0 \mathrm{~kg} / \mathrm{ha})$ in 0-15 cm soil depth at the start of the experiment.

\section{Experimental detail}

The experiment was laid out in Random block design (RBD) with twelve treatments and four replications. Gross and net plot sizes were 5.0 x $3.6 \mathrm{~m}$ and $4.0 \times 2.7 \mathrm{~m}$, respectively.

\section{Agronomic practices}

The graded and healthy seeds of cowpea, Gujarat Cowpea-4 were sown manually in previously opened furrow at the depth of 3 to $5 \mathrm{~cm}$ and at $45 \mathrm{~cm}$ inter-row and $10 \mathrm{~cm}$ intrarow spacing on 7th July, 2007 with recommended seed rate of $25 \mathrm{~kg} \mathrm{ha}^{-1}$. The recommended dose of fertilizer i.e. $20 \mathrm{~kg} \mathrm{~N}$ $\mathrm{ha}^{-1}$ and $40 \mathrm{~kg} \mathrm{P}_{2} \mathrm{O}_{5} \mathrm{ha}^{-1}$ in the form of urea and single super phosphate applied in furrows just before sowing.

\section{Results and Discussion}

In different weed management treatments it was found that treatments $\mathrm{T}_{10}(2 \mathrm{HW}+2 \mathrm{IC}$ 
at 20 and $40 \mathrm{DAS}$ ), $\mathrm{T}_{2}$ (pendimethalin at 0.5 $\mathrm{kg} \mathrm{ha}^{-1}$ pre- emergence $+1 \mathrm{HW}+1 \mathrm{IC}$ at $25-$ $30 \mathrm{DAS}$ ), $\mathrm{T}_{5}$ (quizalofop-ethyl at $0.04 \mathrm{~kg} \mathrm{ha}^{-1}$ post-emergence at 20-25 DAS + $1 \mathrm{HW}+1 \mathrm{IC}$ at $40-45 \mathrm{DAS}$ ) and $\mathrm{T}_{8}$ (imazethapyr at 0.075 $\mathrm{kg} \mathrm{ha}^{-1}$ as post-emergence at 20-25 DAS +1 $\mathrm{HW}+1$ IC at 40-45 DAS) were found very effective to control all three groups sedges, monocots and dicots as it shown in table 1 and at par with weed free treatment. Lowest weed index (WI) were recorded in treatments $\mathrm{T}_{10}(2 \mathrm{HW}+2 \mathrm{IC}$ at 20 and $40 \mathrm{DAS})$ followed by $\mathrm{T}_{2}$ (pendimethalin at $0.5 \mathrm{~kg} \mathrm{ha}{ }^{-1}$ preemergence $+1 \mathrm{HW}+1 \mathrm{IC}$ at 25-30 DAS), $\mathrm{T}_{5}$ (quizalofop-ethyl at $0.04 \mathrm{~kg} \mathrm{ha}^{-1}$ postemergence at 20-25 DAS $+1 \mathrm{HW}+1 \mathrm{IC}$ at 40-45 DAS) and $\mathrm{T}_{8}$ (imazethapyr at $0.075 \mathrm{~kg}$ $\mathrm{ha}^{-1}$ as post-emergence at 20-25 DAS $+1 \mathrm{HW}$ +1 IC at 40-45 DAS) $0.89,8.14,9.63$ and 10.95 per cent respectively. The treatment $T_{12}$ (weedy check) recorded the highest weed index (44.57) as compared to all other treatments (Figure 1).

Table.1 Effect of different treatments on number of weeds (group wise) per $\mathrm{m}^{2}$ recorded at 60 DAS and at harvest

\begin{tabular}{|c|c|c|c|c|c|c|c|}
\hline \multirow{2}{*}{\multicolumn{2}{|c|}{ Treatments }} & \multicolumn{2}{|c|}{ Sedge per $\mathbf{m}^{2}$} & \multicolumn{2}{|c|}{ Monocot per $\mathrm{m}^{2}$} & \multicolumn{2}{|c|}{ Dicot per $\mathbf{m}^{2}$} \\
\hline & & 60DAS & At & 60DAS & $\begin{array}{c}\text { At } \\
\text { harvest }\end{array}$ & 60DAS & At \\
\hline & $\begin{array}{l}\text { Fluchloralin @ } 0.6 \mathrm{~kg} \mathrm{ha}^{-1} \mathrm{PRE}+1 \mathrm{HW}+1 \\
\text { IC at 25-30 DAS }\end{array}$ & $\begin{array}{c}4.16 \\
(17.31)\end{array}$ & $\begin{array}{c}4.28 \\
(18.28)\end{array}$ & $\begin{array}{c}4.94 \\
(24.43)\end{array}$ & $\begin{array}{c}5.08 \\
(25.81)\end{array}$ & $\begin{array}{c}4.87 \\
(23.72)\end{array}$ & $\begin{array}{c}5.02 \\
(25.18)\end{array}$ \\
\hline $\mathrm{T}_{2}$ & $\begin{array}{l}\text { Pendimethalin @ } 0.5 \mathrm{~kg} \mathrm{ha}^{-1} \mathrm{PRE}+1 \mathrm{HW}+1 \\
\text { IC at 25-30 DAS }\end{array}$ & $\begin{array}{c}1.54 \\
(2.36)\end{array}$ & $\begin{array}{l}1.91 \\
(3.66)\end{array}$ & $\begin{array}{c}3.28 \\
(10.77)\end{array}$ & $\begin{array}{c}3.29 \\
(10.82)\end{array}$ & $\begin{array}{c}3.28 \\
(10.77)\end{array}$ & $\begin{array}{c}3.73 \\
(13.91)\end{array}$ \\
\hline $\mathrm{T}_{3}$ & $\begin{array}{l}\text { Quizalofop-ethyl @ } 0.04 \mathrm{~kg} \mathrm{ha}^{-1} \text { POST at } \\
20-25 \text { DAS }\end{array}$ & $\begin{array}{c}4.90 \\
(23.96)\end{array}$ & $\begin{array}{c}5.20 \\
(26.99)\end{array}$ & $\begin{array}{c}5.09 \\
(25.93)\end{array}$ & $\begin{array}{c}5.24 \\
(27.41)\end{array}$ & $\begin{array}{c}5.10 \\
(25.96)\end{array}$ & $\begin{array}{c}5.45 \\
(29.70) \\
\end{array}$ \\
\hline $\mathrm{T}_{4}$ & $\begin{array}{l}1 \text { IC at 8-10DAS + Quizalofop-ethyl } \\
@ 0.04 \mathrm{~kg} \mathrm{ha}^{-1} \text { POST at 20-25 DAS }\end{array}$ & $\begin{array}{c}4.82 \\
(23.18)\end{array}$ & $\begin{array}{c}5.11 \\
(26.11)\end{array}$ & $\begin{array}{c}5.02 \\
(25.20)\end{array}$ & $\begin{array}{c}5.14 \\
(26.45)\end{array}$ & $\begin{array}{c}5.04 \\
(25.38)\end{array}$ & $\begin{array}{c}5.30 \\
(28.12)\end{array}$ \\
\hline $\mathrm{T}_{5}$ & $\begin{array}{l}\text { Quizalofop-ethyl @ } 0.04 \mathrm{~kg} \mathrm{ha}^{-1} \text { POST at } \\
20-25 \text { DAS +1HW + 1 IC at 40-45 DAS }\end{array}$ & $\begin{array}{l}1.72 \\
(2.95)\end{array}$ & $\begin{array}{c}1.99 \\
(3.97)\end{array}$ & $\begin{array}{c}3.32 \\
(10.99)\end{array}$ & $\begin{array}{c}3.39 \\
(11.48)\end{array}$ & $\begin{array}{c}3.35 \\
(11.24)\end{array}$ & $\begin{array}{c}3.77 \\
(14.21)\end{array}$ \\
\hline $\mathrm{T}_{6}$ & $\begin{array}{l}\text { Imazethapyr @ } 0.075 \mathrm{~kg} \mathrm{ha}^{-1} \\
\text { POST at 20-25DAS }\end{array}$ & $\begin{array}{c}5.55 \\
(30.77)\end{array}$ & $\begin{array}{c}5.57 \\
(30.97)\end{array}$ & $\begin{array}{c}5.14 \\
(26.45)\end{array}$ & $\begin{array}{c}5.37 \\
(28.86)\end{array}$ & $\begin{array}{c}5.17 \\
(26.68)\end{array}$ & $\begin{array}{c}5.62 \\
(31.53)\end{array}$ \\
\hline & $\begin{array}{l}1 \mathrm{IC} \text { at } 8-10 \mathrm{DAS}+\text { Imazethapyr @ } 0.075 \mathrm{~kg} \\
\mathrm{ha}^{-1} \text { POST at 20-25 DAS }\end{array}$ & $\begin{array}{c}4.76 \\
(22.65)\end{array}$ & $\begin{array}{c}4.91 \\
(24.18)\end{array}$ & $\begin{array}{c}4.98 \\
(24.85)\end{array}$ & $\begin{array}{c}5.12 \\
(26.21)\end{array}$ & $\begin{array}{c}4.96 \\
(24.60)\end{array}$ & $\begin{array}{c}5.11 \\
(26.13)\end{array}$ \\
\hline & $\begin{array}{l}\text { Imazethapyr@0.075 kg ha }{ }^{-1} \text { POST at 20-25 } \\
\text { DAS +1 HW + } 1 \text { IC at 40-45 DAS }\end{array}$ & $\begin{array}{c}1.78 \\
(3.18)\end{array}$ & $\begin{array}{c}2.04 \\
(4.18)\end{array}$ & $\begin{array}{c}3.34 \\
(11.18)\end{array}$ & $\begin{array}{c}3.42 \\
(11.71)\end{array}$ & $\begin{array}{c}3.40 \\
(11.56)\end{array}$ & $\begin{array}{c}3.79 \\
(14.42)\end{array}$ \\
\hline & $1 \mathrm{HW}+1 \mathrm{IC}$ at $20 \mathrm{DAS}$ & $\begin{array}{c}3.95 \\
(15.60) \\
\end{array}$ & $\begin{array}{c}4.18 \\
(17.47) \\
\end{array}$ & $\begin{array}{c}4.87 \\
(23.71) \\
\end{array}$ & $\begin{array}{c}4.91 \\
(24.18)\end{array}$ & $\begin{array}{c}4.78 \\
(22.84)\end{array}$ & $\begin{array}{c}4.96 \\
(24.65)\end{array}$ \\
\hline & $2 \mathrm{HW}+2 \mathrm{IC}$ at 20 and $40 \mathrm{DAS}$ & $\begin{array}{c}1.28 \\
(1.65)\end{array}$ & $\begin{array}{c}1.38 \\
(1.92)\end{array}$ & $\begin{array}{c}2.91 \\
(8.46)\end{array}$ & $\begin{array}{c}3.15 \\
(9.93)\end{array}$ & $\begin{array}{c}3.07 \\
(9.47)\end{array}$ & $\begin{array}{c}3.35 \\
(11.25)\end{array}$ \\
\hline & Weed free & $\begin{array}{l}0.000 \\
(0.00)\end{array}$ & $\begin{array}{l}0.000 \\
(0.00)\end{array}$ & $\begin{array}{l}0.000 \\
(0.00)\end{array}$ & $\begin{array}{l}0.000 \\
(0.00)\end{array}$ & $\begin{array}{l}0.000 \\
(0.00)\end{array}$ & $\begin{array}{l}0.000 \\
(0.00)\end{array}$ \\
\hline & Weedy check & $\begin{array}{c}6.16 \\
(38.00)\end{array}$ & $\begin{array}{c}6.40 \\
(41.02)\end{array}$ & $\begin{array}{c}6.24 \\
(38.93)\end{array}$ & $\begin{array}{c}6.59 \\
(43.52)\end{array}$ & $\begin{array}{c}6.11 \\
(37.42)\end{array}$ & $\begin{array}{c}6.35 \\
(40.38)\end{array}$ \\
\hline & S.Em. \pm & 0.1962 & 0.24 & 0.16 & 0.19 & 0.21 & 0.17 \\
\hline & C.D. at $5 \%$ & 0.5649 & 0.70 & 0.48 & 0.56 & 0.62 & 0.50 \\
\hline & C.V.\% & 11.59 & 13.61 & 8.18 & 9.36 & 10.55 & 8.05 \\
\hline
\end{tabular}

Square root transformation $(\sqrt{\mathrm{X}})$

Figures in parenthesis are retransformed values 
Table.2 Effect of different treatments on grain and stover yield of cowpea

\begin{tabular}{|c|c|c|}
\hline Treatments & $\begin{array}{c}\text { Grain yield }(\mathrm{kg} \\
\left.\text { ha }^{-1}\right)\end{array}$ & $\begin{array}{c}\text { Stover yield } \\
\left(\mathbf{k g ~ h a}^{-1}\right)\end{array}$ \\
\hline Fluchloralin@0.6 kg ha' ${ }^{-1} \mathrm{PRE}+1 \mathrm{HW}+1$ IC at $25-30 \mathrm{DAS}$ & 1262.73 & 1481.48 \\
\hline Pendimethalin @ $0.5 \mathrm{~kg} \mathrm{ha}^{-1} \mathrm{PRE}+1 \mathrm{HW}+1$ IC at 25-30 DAS & 1465.50 & 1921.29 \\
\hline Quizalofop-ethyl @ $0.04 \mathrm{~kg} \mathrm{ha}^{-1}$ POST at 20-25 DAS & 1203.70 & 1435.18 \\
\hline $\begin{array}{l}1 \text { IC at 8-10DAS + Quizalofop-ethyl } \\
@ 0.04 \mathrm{~kg} \mathrm{ha}^{-1} \text { POST at 20-25 DAS }\end{array}$ & 1226.15 & 1456.01 \\
\hline $\begin{array}{l}\text { Quizalofop-ethyl @ } 0.04 \mathrm{~kg} \mathrm{ha}^{-1} \mathrm{POST} \text { at } 20-25 \mathrm{DAS}+1 \mathrm{HW}+1 \mathrm{IC} \text { at } \\
40-45 \text { DAS }\end{array}$ & 1441.66 & 1900.46 \\
\hline Imazethapyr @ $0.075 \mathrm{~kg} \mathrm{ha}^{-1}$ POST at 20-25DAS & 1174.07 & 1348.61 \\
\hline 1 IC at 8-10DAS + Imazethapyr @ $0.075 \mathrm{~kg} \mathrm{ha}^{-1}$ POST at 20-25 DAS & 1256.02 & 1479.16 \\
\hline $\begin{array}{l}\text { Imazethapyr @ } 0.075 \mathrm{~kg} \mathrm{ha}^{-1} \text { POST at 20-25 DAS }+1 \mathrm{HW}+1 \mathrm{IC} \text { at } \\
\text { 40-45 DAS }\end{array}$ & 1422.22 & 1872.68 \\
\hline $1 \mathrm{HW}+1 \mathrm{IC}$ at $20 \mathrm{DAS}$ & 1287.04 & 1493.06 \\
\hline $2 \mathrm{HW}+2 \mathrm{IC}$ at 20 and $40 \mathrm{DAS}$ & 1581.02 & 1981.48 \\
\hline Weed free & 1595.37 & 2096.53 \\
\hline Weedy check & 884.26 & 1002.31 \\
\hline S.Em. \pm & 95.55 & 118.70 \\
\hline C.D. at $5 \%$ & 275.09 & 341.75 \\
\hline C.V. \% & 14.52 & 14.62 \\
\hline
\end{tabular}

Figure.1 Effect of different treatments on weed index (WI), and weed control index (WCI)

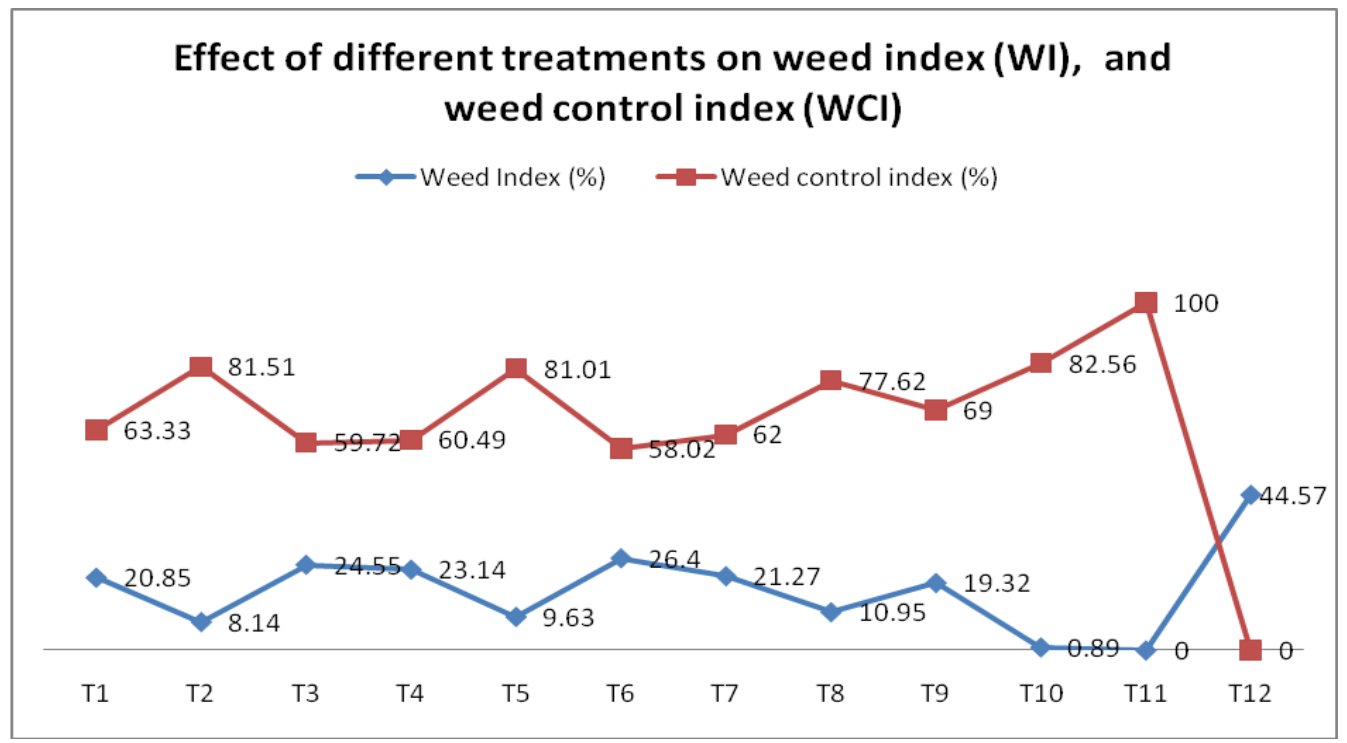


Treatment $\mathrm{T}_{11}$ (weed free) with 100 per cent weed control index (WCI) was closely followed by treatments $\mathrm{T}_{10}(2 \mathrm{HW}+2 \mathrm{IC}$ at 20 and $40 \mathrm{DAS}$ ), $\mathrm{T}_{2}$ (pendimethalin at $0.5 \mathrm{~kg}$ $\mathrm{ha}^{-1}$ pre-emergence $+1 \mathrm{HW}+1 \mathrm{IC}$ at $25-30$ DAS), $T_{5}$ (quizalofop- ethyl at $0.04 \mathrm{~kg} \mathrm{ha}^{-1}$ post-emergence at 20-25 DAS + $1 \mathrm{HW}+1 \mathrm{IC}$ at $40-45$ DAS) and $\mathrm{T}_{8}$ (imazethapyr at 0.075 $\mathrm{kg} \mathrm{ha}^{-1}$ as post-emergence at 20-25 DAS + 1 $\mathrm{HW}+1$ IC at 40-45 DAS) having weed control index of 82.56, 81.51, 81.01 and 77.62 per cent, respectively.

Grain yield of cowpea was significantly influenced due to different weed control treatments tried in this experiment. The treatment $\mathrm{T}_{11}$ (weed free) established its superiority by recoding significantly higher grain yield $1595.37 \mathrm{~kg} \mathrm{ha}^{-1}$ (Table 2). However, in different integrated treatments, it was found statistically at par with treatments $\mathrm{T}_{10}(2 \mathrm{HW}+2 \mathrm{IC}$ at 20 and 40 DAS 1581.02 $\mathrm{kg} \mathrm{ha}^{-1}$ ), $\mathrm{T}_{2}$ (pendimethalin at $0.5 \mathrm{~kg} \mathrm{ha}^{-1}$ preemergence $+1 \mathrm{HW}+1$ IC at 25-30 DAS $1465.50 \mathrm{~kg} \mathrm{ha}^{-1}$ ), $\mathrm{T}_{5}$ (quizalofop- ethyl at 0.04 $\mathrm{kg} \mathrm{ha}^{-1}$ post-emergence at 20-25 DAS +1 $\mathrm{HW}+1$ IC at 40-45 DAS $1441.66 \mathrm{~kg} \mathrm{ha}^{-1}$ ) and $\mathrm{T}_{8}$ (imazethapyr at $0.075 \mathrm{~kg} \mathrm{ha}^{-1}$ as postemergence at 20-25 DAS + $1 \mathrm{HW}+1 \mathrm{IC}$ at 40-45 DAS $1422.22 \mathrm{~kg} \mathrm{ha}^{-1}$ ), in descending order. Single application of herbicide not found sufficient to control weed and significantly yield loss in cowpea. Cowpea farmer can replace 20-25 DAS $1 \mathrm{HW}+1$ IC cultural practices with suitable herbicide to get same yield. Stover yield also show same pattern as grain yield. Results obtained are in conformity with the findings of Madukwe et al., (2012) and Gupta et al., (2016).
In conclusion, integrated weed management is better approach for reduce the yield losses in cowpea due to weeds. Cowpea farmer can replace 20-25 DAS (1 HW + 1 IC) cultural practices with suitable herbicide to get same yield.

\section{Acknowledgement}

The author is thankful to Dr. L.V. Lakkad, Ex Professor Department of Agronomy, College of Agriculture, JAU, Junagadh for providing land, inputs and other facilities required for conducting the above experiment.

\section{References}

Andargie, M, Remy, P., Gowda, B., Muluvi, G., Timko, M. 2011. Construction of a SSRbased genetic map and identification of QTL for domestication traits using recombinant inbred lines from a cross between wild and cultivated cowpea [Vigna unguiculata (L.) Walp.]. Mol. Breed., 28: 413-420.

Gupta, K.C., Gupta, A.K., and Saxena, R. 2016. Weed management in cowpea [Vigna unguiculata (L.) Wasp.] under rainfed conditions. Int. J. Agri. Sci., 12(2): 238240.

Madukwe, D.K., Ogbuehi, H.C. and Onuh, M.O. 2012. Effects of weed control methods on the growth and yield of cowpea [Vigna unguiculata (L.) Walp] under rain-fed conditions of owerri. Am.-Eur. J. Agri. Environ. Sci., 12(11): 1426-1430.

Mohanty, S., Satyasai, K.J. 2015. Feeling the Pulse Indian Pulses Sector. NABARD Rural Pulse.

\section{How to cite this article:}

Pravindra Kumar and Raghuveer Singh. 2017. Integrated Weed Management in Cowpea [Vigna unguiculata (L.)Wasp.] under Rainfed Conditions. Int.J.Curr.Microbiol.App.Sci. 6(3): 97-101. doi: https://doi.org/10.20546/ijcmas.2017.603.010 